\title{
Intraoperative monitoring of visual function
}

\author{
Hugues Duffau
}

Received: 14 July 2011 / Accepted: 19 July 2011 /Published online: 24 August 2011

(C) Springer-Verlag 2011

Due to the excellent functional outcomes reported in the study published in this issue of Acta Neurochirurgica by Benedicic et al. [1], "Intraoperative monitoring of the visual function using cortical potentials after electrical epidural stimulation of the optic nerve", the authors have first of all to be congratulated.

Indeed, they recorded the intraoperative visual evoked potential after electrical epidural stimulation of the optic nerve in 11 patients who underwent surgical resection for a skull base meningioma. They observed that P20 and N30 amplitude changes were related to the manipulation of ON during anterior skull base tumor removal. Interestingly, there was no immediate postoperative visual worsening, although the rate of postsurgical visual deterioration is usually higher in the literature, up to $10 \%$ in patients with no presurgical deficit. It is nonetheless worth noting that Benedicic et al. have already reported the stability and reproducibility of cortical potentials after electrical epidural stimulation of the ON. In addition, several other authors have previously performed intraoperative monitoring of visual function in patients with tumors along the visual pathways (from ON to optic radiation), using different techniques such as flash visual evoked potentials [2] or direct subcortical electrostimulation of the optic radiation in awake patients [3]. Therefore, from a methodological point of view, no new

H. Duffau $(\bowtie)$

Department of Neurosurgery, Gui de Chauliac Hospital, Institute for Neurosciences of Montpellier,

Montpellier University Medical Center and INSERM U1051,

80 avenue Augustin Fliche,

34295 Montpellier Cedex 5, France

e-mail: h-duffau@chu-montpellier.fr data are described here. The results presented by Benedicic et al. using cortical potentials should now be reproduced and validated by other teams.

Furthermore, it is not really surprising to observe P20 and N30 amplitude changes during manipulation of ON. Using the recording of ON-evoked potentials, Kikuchi et al. already noted attenuation of amplitude as a response to ON manipulation [4]. Thus, the actual contribution of the present series would have been to demonstrate that the new technique introduced by the authors was able to predict postoperative deterioration. Unfortunately, the authors failed to identify any correlation between intrasurgical changes of cortical potentials and newly acquired postoperative visual worsening. It would have been helpful to focus more on this point. Indeed, the aim of oncological surgery is to optimize the benefit-to-risk ratio of the surgical procedure, that is, to increase the extent of resection while preserving the quality of life. In the present study, no oncological considerations have been detailed: the extent of tumor removal and the long-term follow-up were not described. Thus, because P20 and N30 amplitude changes were related to the manipulation of $\mathrm{ON}$ or chiasma during anterior skull base tumor removal, one could think that the authors stopped the resection earlier. As a consequence, the question is to know whether it was justified to interrupt the meningiomal removal according to the results provided by these online cortical potentials or whether the patients would have been able to recover even if the authors had pursued the resection in spite of the electrophysiological changes.

In summary, despite the rigorous methodology and the favorable functional outcomes here described, on the basis of this small series reporting a negative result (i.e., no correlation between intrasurgical modifications of cortical potentials and newly acquired postoperative 
visual worsening), it is currently impossible to reach a conclusion regarding the actual impact of this method on the functional/oncological balance and thus about the need to use it (or not) during surgery for skull base tumors. Prospective multicenter studies should be performed to assess the real contribution of such intraoperative neurophysiological monitoring to prevent the loss of visual function during surgery in the parasellar region.

\section{Conflicts of interest None.}

\section{References}

1. Benedicic M, Bosnjak R (in press) Intraoperative monitoring of the visual function using cortical potentials after electrical epidural stimulation of the optic nerve. Acta Neurochir (Wien)

2. Cedzich C, Schramm J (1990) Monitoring of flash visual evoked potentials during neurosurgical operations. Int Anesthesiol Clin 28:165-169

3. Duffau H, Velut S, Mitchell MC, Gatignol P, Capelle L (2004) Intraoperative mapping of the subcortical visual pathways using direct electrical stimulations. Acta Neurochir (Wien) 146:265-269

4. Kikuchi Y, Sasaki T, Matsumoto M, Oikawa T, Itakura T, Kodama N (2005) Optic nerve evoked potentials elicited by electrical stimulation. Neurol Med Chir (Tokyo) 45:349-355 